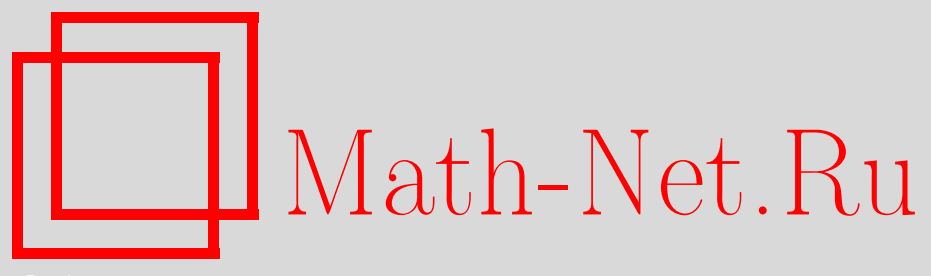

Е. В. Бурнаев, Задача о разладке для пуассоновского процесса в обобщенной байесовской постановке, $У М H$, 2007, том 62, выпуск 4, 151-152

DOI: https://doi.org/10.4213/rm6810

Использование Общероссийского математического портала Math-Net.Ru подразумевает, что вы прочитали и согласны с пользовательским соглашением http://www . mathnet.ru/rus/agreement

Параметры загрузки:

IP : 54.197 .217 .227

26 апреля 2023 г., 03:15:03

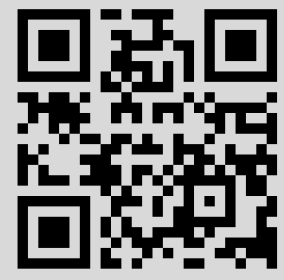




\section{Задача о разладке для пуассоновского процесса в обобщенной байесовской постановке}

\section{Е. В. Бурнаев}

1. Предполагается, что на некотором вероятностном пространстве $(\Omega, \mathscr{F}, \mathrm{P})$ наблюдается случайный процесс $X=\left(X_{t}\right)_{t \geqslant 0}$, имеющий вид $X_{t}=\int_{0}^{t} I(s<\theta) d N_{s}^{\lambda_{0}}+$ $\int_{0}^{t} I(s \geqslant \theta) d N_{s-\theta}^{\lambda_{1}}, \quad X_{0}=0$, где $N^{\lambda_{0}}=\left(N_{t}^{\lambda_{0}}\right)_{t \geqslant 0}$ и $N^{\lambda_{1}}=\left(N_{t}^{\lambda_{1}}\right)_{t \geqslant 0}$ - пуассоновские процессы с интенсивностями $\lambda_{0}$ и $\lambda_{1}$ соответственно, $I(A)$ - индикатор события $A$. Неслучайный момент $\theta \in[0, \infty]$ интерпретируется как момент появления "разладки" (по терминологии [1], [2]) в ходе наблюдения за процессом $X$ : при $t<\theta$ наблюдаемый процесс $X_{t}=N_{t}^{\lambda_{0}}$, а при $t \geqslant \theta$ процесс $X_{t}=N_{\theta}^{\lambda_{0}}+N_{t-\theta}^{\lambda_{1}}$. Задача будет рассматриваться в предположении, что $\lambda_{1}<\lambda_{0}$.

Будем через $\tau=\tau(\omega)$ обозначать момент подачи сигнала о появлении "разладки", т. е. пусть $\tau$ есть марковский момент со значениями в $[0, \infty]$ относительно потока $\left(\mathscr{F}_{t}^{X}\right)_{t \geqslant 0}$, где $\mathscr{F}_{t}^{X}=\sigma\left\{\omega: X_{s}, s \leqslant t\right\}, t \geqslant 0$.

В настоящей работе доказывается оптимальность статистики Ширяева (введенной в [1], [2]) в смысле минимизации обобщенного байесовского критерия (рассмотренного в $[3])$.

Пусть $\mathrm{P}_{t}$ - распределение вероятностей процесса $X$ в предположении, что момент разладки $\theta=t$. Распределение $\mathrm{P}_{\infty}$ отвечает случаю отсутствия разладки $(\theta=\infty)$, $\mathrm{P}_{0}$ соответствует случаю наличия разладки в момент $\theta=0$. Обозначим $\mathscr{M}_{T}=\{\tau$ : $\left.\mathrm{E}_{\infty} \tau=T\right\}-$ класс моментов остановки $\tau$, для которых среднее время $\mathrm{E}_{\infty} \tau$ до подачи (ложной) тревоги равно $T$.

Качество системы наблюдения, идентифицируемой с выбором момента $\tau \in \mathscr{M}_{T}$, будем оценивать величиной $B(T ; \tau)=\frac{1}{T} \int_{0}^{\infty} \mathrm{E}_{\theta}(\tau-\theta)^{+} d \theta$, где $x^{+}=\max (0, x)$. Момент $\tau_{T}^{*} \in \mathscr{M}_{T}$ естественно называть оптимальным (в обобщенном байесовском смысле), если $B(T)=B\left(T ; \tau_{T}^{*}\right)=\inf _{\tau \in \mathscr{M}_{T}} B(T ; \tau)$. В используемом байесовском критерии параметр $\theta$ можно интерпретировать как обобщенную случайную величину с "равномерным" распределением на $[0, \infty)$.

Обозначим через $\psi=\left(\psi_{t}\right)_{t \geqslant 0}$ процесс Ширяева, имеющий вид $\psi_{t}=\int_{0}^{t} \frac{L_{t}}{L_{s}} d s$, где $L_{t}=\frac{d\left(\mathrm{P}_{0} \mid \mathscr{F}_{t}^{X}\right)}{d\left(\mathrm{P}_{\infty} \mid \mathscr{F}_{t}^{X}\right)}=\exp \left(\log \left(\frac{\lambda_{1}}{\lambda_{0}}\right) X_{t}-\left(\lambda_{1}-\lambda_{0}\right) t\right)-$ производная Радона-Никодима сужения $\mathrm{P}_{0} \mid \mathscr{F}_{t}^{X}$ меры $\mathrm{P}_{0}$ относительно сужения $\mathrm{P}_{\infty} \mid \mathscr{F}_{t}^{X}$ меры $\mathrm{P}_{\infty}[1]$.

Пусть $\rho=\frac{1}{2}\left(\frac{\lambda_{1}-\lambda_{0}}{\sqrt{\lambda_{0}}}\right)^{2}, \beta=\frac{1}{6}\left(\frac{\lambda_{1}-\lambda_{0}}{\sqrt{\lambda_{0}}}\right)^{3},-\operatorname{Ei}(-x)=\int_{x}^{+\infty} \frac{e^{-t}}{t} d t \quad(x>0)-$ интегральная показательная функция [4], $\mathbb{C}=0.577 \ldots-$ константа Эйлера. Выполняется следующая теорема.

Теорема. При каждом $T>0$ в классе $\mathscr{M}_{T}$ существует оптималъный момент $\tau_{T}^{*}$ такой, что $\tau_{T}^{*}=\inf \left\{t \geqslant 0: \psi_{t} \geqslant T\right\}$. При этом обобщенный байесовский риск $B(T)=V(0 ; T) / T$, где $V(x ; T)$ - решение дифференииально-разностного уравнения

$$
\begin{gathered}
\left(1-\left(\lambda_{1}-\lambda_{0}\right) x\right) V^{\prime}(x ; T)+\lambda_{0}\left(V\left(\frac{\lambda_{1}}{\lambda_{0}} x ; T\right)-V(x ; T)\right)=-x, \\
V(0 ; T)<\infty, \quad V(T ; T)=0 .
\end{gathered}
$$

Работа частично поддержана аналитической ведомственной целевой программой РНП.2.2.1.1.2467, программой "Ведущие научные школы” (грант НШ-5379.2006.1), программой фундаментальных исследований Президиума РАН 15, грантом РГНФ 06-02-91821 a/G, грантом РФФИ 05-01-00944. 
Если $\lambda_{0} \rightarrow \infty u \lambda_{1} \rightarrow \infty$ так, что для некоторой фиксированной константы $C<0$ выполняется равенство $\lambda_{1}=\lambda_{0}+\sqrt{\lambda_{0}} C$, mо $B(T)=B_{0}(T)+\varepsilon B_{1}(T)+\cdots$, где малый параметр $\varepsilon=1 / \sqrt{\lambda_{0}}$,

$$
\begin{aligned}
& B_{0}(T)=\frac{1}{\rho}\left[e^{\frac{1}{\rho T}}\left(-\operatorname{Ei}\left(-\frac{1}{\rho T}\right)\right)-\left(1-\frac{1}{\rho T} \int_{0}^{\infty} \frac{e^{-t} \log (1+\rho T \cdot t)}{t} d t\right)\right], \\
& B_{1}(T)=\frac{\beta}{\rho^{2}}\left[\frac{3}{\rho T} \int_{0}^{\infty} \frac{e^{-t} \log (1+\rho T \cdot t)}{t} d t+e^{\frac{1}{\rho T}} \operatorname{Ei}\left(-\frac{1}{\rho T}\right) \cdot\left(\frac{1}{2 \rho T}-1\right)-\frac{5}{2}\right],
\end{aligned}
$$

при этом

$$
\begin{aligned}
& B_{0}(T)= \begin{cases}\frac{1}{\rho}\left[\frac{\rho T}{2}+O\left((\rho T)^{2}\right)\right] & \text { nри } T \rightarrow 0, \\
\frac{1}{\rho}\left[\log (\rho T)-1-\mathbb{C}+O\left(\frac{\log ^{2} \rho T}{\rho T}\right)\right] & \text { nри } T \rightarrow \infty,\end{cases} \\
& B_{1}(T)= \begin{cases}\frac{\beta}{\rho^{2}}\left[\frac{(\rho T)^{3}}{2}+O\left((\rho T)^{4}\right)\right] & \text { nрu } T \rightarrow 0, \\
\frac{\beta}{\rho^{2}}\left[\log (\rho T)-\frac{5}{2}-\mathbb{C}+O\left(\frac{\log ^{2} \rho T}{\rho T}\right)\right] & \text { при } T \rightarrow \infty .\end{cases}
\end{aligned}
$$

2. Теорема доказывается следующим образом.

Прежде всего, необходимо заметить, что лемма 2.1 статьи [3] может быть доказана и в случае пуассоновского процесса. Из этой леммы следует, что $B(T)=$ $\inf _{\tau \in \mathscr{M}_{T}} \frac{1}{T} \mathrm{E}_{\infty} \int_{0}^{\tau} \psi_{u} d u$.

Далее, применяя метод множителей Лагранжа, описанный в [3; раздел 4], и технику из [1; раздел 24], можно доказать, что оптимальным моментом остановки будет момент $\tau_{T}^{*}=\inf \left\{t \geqslant 0: \psi_{t} \geqslant T\right\}$, при этом риск $B(T)=V(0 ; T) / T$, где $V(x ; T)-$ решение уравнения (1).

Разлагая решение уравнения (1) по малому параметру $\varepsilon$ при $\lambda_{1} \rightarrow \infty$ и $\lambda_{0} \rightarrow \infty$ $\left(\lambda_{1}=\lambda_{0}+\sqrt{\lambda_{0}} C\right.$ для некоторой фиксированной константы $\left.C<0\right)$, получаем, что $V(x ; T)=V_{0}(x ; T)+\varepsilon V_{1}(x ; T)+\cdots$, где $V_{0}(x ; T)$ и $V_{1}(x ; T)$ - решения уравнений

$$
V_{0}^{\prime}(x ; T)+\rho x^{2} V_{0}^{\prime \prime}(x ; T)=-x, \quad V_{0}(0 ; T)<\infty, \quad V_{0}(T ; T)=0
$$

и

$$
V_{1}^{\prime}(x ; T)+\rho x^{2} V_{1}^{\prime \prime}(x ; T)=-\beta x^{3} V_{0}^{\prime \prime \prime}(x ; T), \quad V_{1}(0 ; T)<\infty, \quad V_{1}(T ; T)=0
$$

соответственно. Отсюда следует, что $B_{0}(T)=V_{0}(0 ; T) / T$ и $B_{1}(T)=V_{1}(0 ; T) / T$. Заметим, что, как и следовало ожидать, функция $B_{0}(T)$ совпадает с обобщенной байесовской функцией риска в задаче о разладке для броуновского движения, рассмотренной в [3].

Автор благодарен А.Н. Ширяеву, инициировавшему написание данной заметки.

\section{Список литературы}

[1] G. Peskir, A. N. Shiryaev, Optimal stopping and free-boundary problems, Lectures Math. ETH Zurich, Birkhäuser, Basel, 2006. [2] А. Н. Ширяев, Статистический последовательнъй анализ. Оптимальные правила остановки, Наука, М., 1976. [3] Е. A. Feinberg, A. N. Shiryaev, Statist. Decisions, 24 (2006), 1001-1025. [4] И. С. Градштейн, И. М. Рыжик, Таблицы интегралов, сумм, рядов и произведений, Физматгиз, М., 1963.

Е. В. Бурнаев (Е. V. Burnaev)

Московский физико-технический институт (государственный университет)

E-mail: drazil@list.ru
Представлено А. В. Булинским Принято редколлегией 30.03.2007 